\title{
El achiote Bixa orellana $L$. como posible alternativa productiva para el Departamento del Meta
}

\section{The achiote Bixa orellana $L$. as productive possible alternative to Meta}

\section{Department}

\author{
Quiñones Bravo Ximena ${ }^{1}$ y Yunda Romero Myriam Constanza ${ }^{2}$ \\ ${ }^{1}$ Ing Agr. Unillanos y ${ }^{2}$ Ing. Agr. Esp. MSc. Docente Unillanos \\ cyunda@unillanos.edu.co
}

Recibido 15 de Enero de 2014, Aceptado 11 de Abril 2014

\section{RESUMEN}

Se realizó un análisis del achiote Bixa orellana L. como posible alternativa productiva de desarrollo industrial para el departamento del Meta, mediante la recopilación de información de investigaciones sobre esta planta y las experiencias de países como Perú, en los cuales el achiote es uno de los principales productos de exportación. En primer lugar, se generó un marco de referencia centrado en las características fenológicas, requerimientos climatológicos, aspectos agronómicos, de procesamiento y mercados. Esta especie amazónica, se encuentra distribuida en 19 de los 32 departamentos del país, se adapta a suelos químicamente pobres, siempre que presente buenas condiciones físicas (texturas franco arenosas) para el desarrollo radicular, las mejores producciones se logran en altitudes menores a los $800 \mathrm{msnm}$, es un buen demandante de agua y para evitar problemas patológicos a nivel de las semillas, es importante no tener humedades relativas por encima del $80 \%$. Los principales insectos que causan daño son los chupadores como los chinches (Leptoglossus zonatus), los masticadores como las hormigas (Atta mexicana) y barrenadores de la capsula (Milgithea melanoleuca). Entre los principales patógenos que pueden afectar el cultivo están los hongos Oidium bixaea, Collectotrichum gloeosporioides, Crinipellis perniciosa, Fusarium sp. y Cercospora bixae. La producción de cápsulas por hectárea puede variar dependiendo de la zona y sobre todo del 
manejo del cultivo, encontrando producciones por hectárea mayores a 5 toneladas y menores de 1 tonelada. En conclusión, la creciente demanda mundial de colorantes naturales, la oferta agroclimatológica del departamento del Meta, la cercanía a la capital como principal mercado para la comercialización de este producto en la cadena productiva de plantas aromáticas y medicinales son oportunidades para el desarrollo económico de esta planta.

Palabras clave: Achiote, Bixa orellana, colorante natural.

\begin{abstract}
An analysis of Bixa orellana L. achiote productive as possible alternative for industrial development Meta was performed by collecting information on this plant research and the experiences of countries like Peru, in which the annatto is one of the main export products. First, a framework focused on phenology, climatic requirements, agronomic, processing and markets was generated. Was found that this amazon species is distributed in 19 of the 32 departments, fits chemically poor soils, provided they present good physical condition (sandy loam texture) for root development, the best yields are achieved at lower altitudes $800 \mathrm{~m}$, is a good user of water and to prevent pathological level seed problems, it is important not to have relative humidities above $80 \%$. The main insects that cause damage are sucking bugs as (Leptoglossus zonatus) chewing like ants (Atta mexicana) and capsule borer (Milgithea melanoleuca). The main pathogens that may affect the crop are fungi Oidium bixaea, Collectotrichum gloeosporioides, Crinipellis pernicious, Fusarium sp. and Cercospora bixae. The capsule production per hectare can vary depending on the area and especially crop management, finding productions per hectare greater than 5 tons and less than 1 ton. In conclusion, the increasing global demand for natural dyes, the Meta offer agroclimatic, proximity to the capital as the main market for the trading of this product and the development of the productive chain of aromatic and medicinal plants are opportunities for the economic development of this plant.
\end{abstract}

Keywords: Achiote, Bixa orellana, natural dye. 


\section{INTRODUCCIÓN}

El achiote Bixa orellana, es una planta de un gran potencial, por su contenido de Bixina en las semillas, usado como colorante natural, el cual se está posicionando en el mercado de los colorantes naturales, con un fuerte incremento de la demanda en los últimos cinco años por parte de los países desarrollados, puesto que es utilizado por diferentes industrias como la textil, cosmética, de alimentos y farmacéutica (Giuliano et al., 2003).

El uso de los colorantes de origen natural se ha incrementado, en el año 2009 tenía un valor estimado de US\$1.450 millones, en la actualidad está alrededor de 40.000 a 50.000 toneladas anuales, y se espera que en el 2015 el valor del mercado mundial pueda llegar a US $\$ 1.6$ billones con un aumento en el futuro de $10 \%$ de su nivel actual. De 2005 a 2009, el mercado mundial de colorantes naturales aumentó casi $35 \%$, su alta demanda se debe a la exigencia en las industrias alimenticias, farmacéuticas, cosméticas, además se cumple con los requerimientos contemplados en las legislaciones de algunos países (Pérez y Rojas, 2010).

En la actualidad tanto consumidores como industriales prefieren la utilización de estos productos de origen natural debido a su biodegradabilidad y baja toxicidad, contrario a lo que ocurre con los colorantes artificiales que pueden contener compuestos químicos que llegan a ser tóxicos (James et al., 2003). El consumidor más allá de satisfacer sus necesidades cuida su salud. En la búsqueda de opciones para responder a las tendencias mundiales se reconoce la exigencia de una oferta de especies vegetales con posibilidades de uso como colorantes naturales, los cuales están contenidos en las semillas del achiote (Bixa orellana L.), y que actualmente tiene buena aceptación en las industrias de cosmetología, alimentos, textil y farmacéuticas.

El achiote es una especie originaria de América tropical, posiblemente del suroeste de la amazonia, puede tener una vida útil de hasta 50 años, presentando su primera cosecha a partir de los 15 a 18 meses, obteniendo rendimientos que 
oscilan entre 800 y $960 \mathrm{~kg} / \mathrm{ha}$, aunque sus producciones pueden llegar hasta $2.500 \mathrm{~kg} / \mathrm{ha}$ en plantaciones con un buen manejo agronómico. Los países líderes en investigación sobre el achiote, con experiencias en su manejo como sistema productivo y quienes a nivel de Latinoamérica se reconocen como los principales exportadores son Perú, México, Costa Rica, El Salvador y Brasil. El principal productor de achiote es Perú con un 35\% de la producción mundial, seguido de Kenia, mientras que Estados Unidos es el principal demandante de achiote en forma de polvo (INIA, 2009).

Los usos de Bixa orellana son muy amplios, se reportan propiedades farmacéuticas por su acción cicatrizante, antioxidante, microbiana y antiparasitaria entre otras (James et al., 2003); su madera se utiliza en la elaboración de muebles, mangos para herramientas y en construcciones; el aceite de la semilla es emoliente y se utiliza en la elaboración de productos cosméticos. El achiote hace parte de la cadena productiva de plantas aromáticas, medicinales y condimentarías, dentro de ésta se cataloga como especie priorizada (teniendo en cuenta las tendencias históricas, presentes, futuras en diferentes mercados y la disponibilidad de oferta actual y potencial), lo cual fundamenta aun más la posibilidad de considerarlo como alternativa productiva para el departamento del Meta.

\section{MORFOLOGÍA DE LA PLANTA}

Es un arbusto o árbol pequeño, perennifolio o caducifolio, de 2 a $5 \mathrm{~m}$ (hasta $10 \mathrm{~m}$ ) de altura, con un diámetro a la altura del pecho de 20 a $30 \mathrm{~cm}$; su sistema radical es pivotante y muy largo; su copa es redondeada y densa, sus hojas simples, alternas, grandes y lustrosas, ovadas, de punta larga en el ápice, con pecíolos delgados y largos, acorazonadas en la base, con puntos notables de color marrón en el envés, de 9 a $19 \mathrm{~cm}$ de largo por 6 a $11 \mathrm{~cm}$ de ancho; el tronco es cilíndrico, las ramas jóvenes son delgadas y la corteza externa es de color café claro, algo fisurada, y se desprende fácilmente en largas tiras; internamente amarilla o 
amarillo-anaranjada, con savia del mismo color, pegajosa y ligeramente amarga (Bonilla, 2009).

Las flores (Figura 1) son compuestas, actinomorfas, de sexualidad hermafrodita, dispuestas en panoja, poseen cáliz de cinco pétalos libres de prefloración imbricada y cinco sépalos; tiene estambres indefinidos, libres en la base, con filamentos filiformes, de color blanco, amarillo y violeta; posee anteras bitecas y de dehiscencia poricida; el ovario es elevado por un ginóforo y adopta la apariencia de ser supero, con rudimentos seminales que varían de 10 a 60 por capsula; unilocular, a veces con falsos tabiques, pluriovulado, estilo filiforme, y con estigma bilobulado; dependiendo de la coloración de la flor, las capsulas son verdes, rojizas o amarillas (Bonilla, 2009).

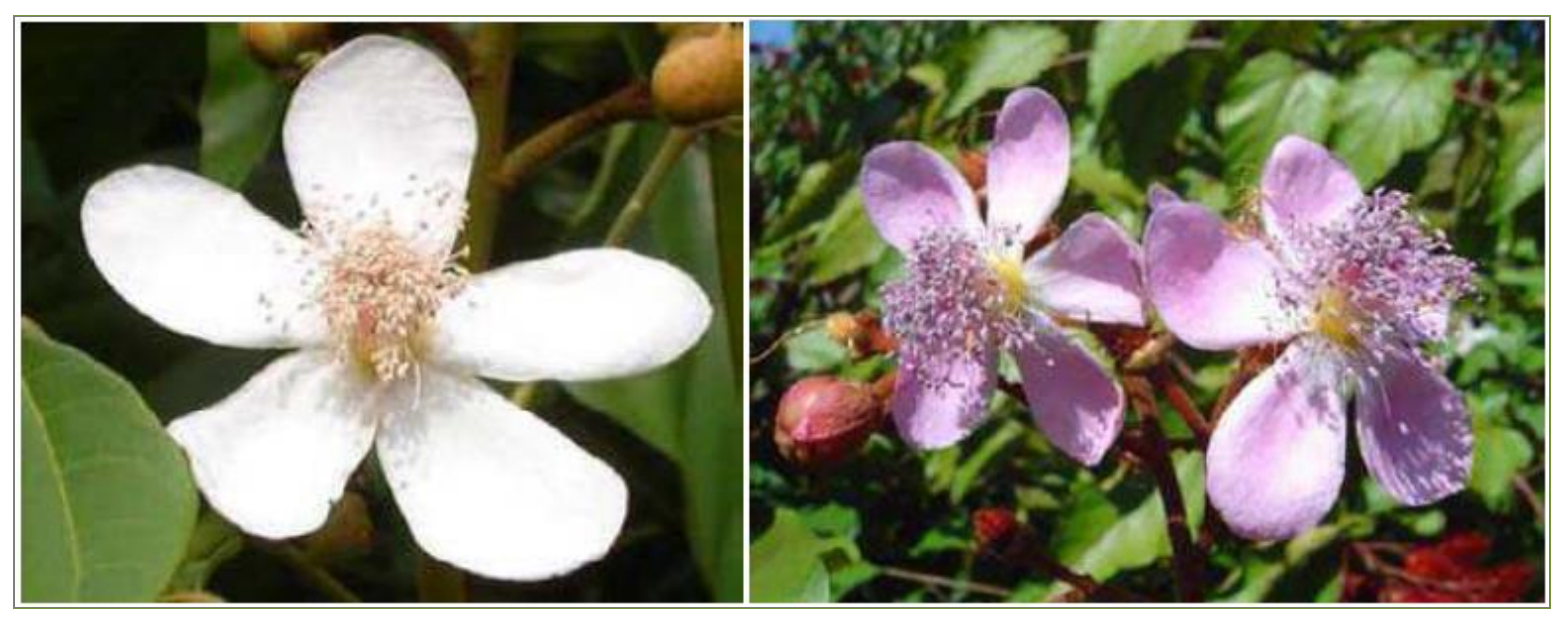

Figura 1. Flores de diferentes colores de Bixa orellana $L$. Tomado de Bonilla, 2009.

El fruto es una cápsula ovoide a globosa, pardo-rojiza, de 3 a $5 \mathrm{~cm}$ de largo por 3 a $4.4 \mathrm{~cm}$ de diámetro, dehiscente por 2 valvas, erizado de pequeños aguijones delgados y blandos hasta de $6 \mathrm{~mm}$, conteniendo aproximadamente 50 semillas, las cuales son rojas casi triangulares algo comprimidas y pequeñas, de 5 a 5.5 $\mathrm{mm}$ de largo por 4 a $5 \mathrm{~mm}$ de ancho, con una testa pulposa de color rojo y albumen carnoso, los cotiledones son planos (Bonilla, 2009). 


\section{CARACTERÍSTICAS FISICOQUÍMICAS DE LA SEMILLA DEL ACHIOTE}

El principal constituyente colorante de la semilla del achiote es la bixina, que se encuentra en la cubierta exterior del fruto, y representa más del $80 \%$ de los pigmentos presentes, lo cual facilita su extracción; sus componentes principales son: resina, materia colorante amarilla denominada Orellina, aceite volátil, aceite graso, y en un $80 \%$ la materia colorante roja conocida como bixina. Según diferentes fuentes, la composición tanto química como nutricional de los pigmentos de la semilla del achiote es muy variada (Tabla 1) (Devia y Saldarriaga, 2003).

Tabla 1. Composición química y nutricional de los pigmentos del achiote

\begin{tabular}{|c|c|c|c|c|c|}
\hline \multicolumn{2}{|c|}{ Composición Química } & \multicolumn{2}{|c|}{$\begin{array}{c}\text { Composición } \\
\text { nutricional }(\mathrm{mg} / 100 \mathrm{~g})\end{array}$} & \multicolumn{2}{|c|}{$\begin{array}{l}\text { Composición de } \\
\text { pigmentos g/ } 100 \mathrm{~g} \text { ) }\end{array}$} \\
\hline Humedad & $8-13 \%$ & Calcio & 7 & Proteínas & $12.3-13.2$ \\
\hline Proteína & $13-14.24 \%$ & Fosforo & 10 & Pectinas & 0.23 \\
\hline Celulosa & $13.8 \%$ & Hierro & 1.4 & Carbohidratos & $39.91-47.9$ \\
\hline Fibra cruda & $18.48 \%$ & Vitamina A & 45 & Ceniza & $5.44-6.92$ \\
\hline Almidones & $11.45 \%$ & Riboflavina & 0.2 & Taninos & $0.33-0.91$ \\
\hline Carbohidratos & $39.91 \%$ & Niacina & 1.46 & Pentosanos & $11.35-14.97$ \\
\hline Ceniza & $4.5-7.97 \%$ & Tiamina & 0.39 & Carotenoides & $1.21-2.3$ \\
\hline Energía & $54 \mathrm{Kcal}$ & $\begin{array}{c}\text { Acido } \\
\text { Ascórbico }\end{array}$ & 12.5 & $\beta$-carotenos & $6.8-11.3 \mathrm{mg}$ \\
\hline
\end{tabular}

Fuente: Tomado de Devia y Saldarriaga, 2003

El principal componente del colorante de la semilla del achiote es la bixina de color rojo oscuro, que es un ácido carotenóico de formula empírica $\mathrm{C}_{25} \mathrm{H}_{30} \mathrm{O}_{4}$, que se presenta como isómero geométrico del tipo cis, pero que puede convertirse a su forma trans, más estable. Es insoluble en agua y ligeramente soluble en cloroformo, aceites vegetales, acetato de etilo y propilenglicol. Al hervir la bixina en una solución de álcali, se forma una molécula de metanol y una sal dipotásica que, por acidificación, produce el ácido dibásico norbixina, $\mathrm{C}_{24} \mathrm{H}_{28} \mathrm{O}_{4}$, pigmento carotenoide soluble en agua (Devia y Saldarriaga, 2003). 


\section{IMPORTANCIA DE LA ESPECIE Bixa Orellana}

\section{Importancia económica}

El achiote presenta una gran demanda y acogida en los países desarrollados por sus diversos usos como colorante en la industria alimenticia, en productos cosméticos, telas y sedas, además de algunos usos medicinales; esto lleva a la necesidad de aumentar la frontera agrícola y de generar un valor agregado sobre el cultivo (Cabrera y Piza, 2010).

\section{Importancia ecológica y ambiental}

La planta de achiote produce anualmente una cobertura de hojarasca, proporcionando una fuerte cantidad de biomasa lo que permite la formación de humus en el suelo, también es fuente productora de oxígeno por formar una masa boscosa. Asimismo, existen reportes del uso como barrera rompe viento, plantada en una sola hilera forma una cortina muy efectiva, también tiene un beneficio ornamental puesto que las flores, conspicuas y frutos espinosos le dan un aspecto muy llamativo (Bonilla, 2009).

\section{Importancia farmacológica}

Debido a la abundante evidencia etnomédica que apoya las potencialidades terapéuticas del achiote, se han realizado múltiples estudios para determinar su actividad biológica (Lourido y Martínez, 2010), a continuación, se describen los más representativos.

- Acción cicatrizante. Se utilizó un extracto alcohólico de Bixa orellana en piel lesionada de conejos en Nueva Zelanda y se logró una recuperación de la lesión total a los 3 días de la aplicación del producto (González et al., 2003). También se empleó una crema al 5 y 10\% en conejos y ratas con heridas quirúrgicas o accidentales y se comprobó un fuerte efecto cicatrizante.

- Acción antioxidante. Se evaluó el efecto de la norbixina en la respuesta al daño del ADN inducido por radiación UV, peróxido de hidrógeno $\left(\mathrm{H}_{2} \mathrm{O}_{2}\right)$ y 
anión superóxido $\left(\mathrm{O}_{2}\right)$ sobre células de Escherichia coli, y se determinó que la norbixina era capaz de proteger a la célula ante estos agentes. La norbixina aumentó la supervivencia de la célula en al menos 10 veces (Júnior et al., 2005). Otros experimentos demostraron que la bixina actuaba como antioxidante interceptando los radicales libre generados por el daño cromosómico inducido por el agente cisplatino (Antunes et al., 2005).

- Acción antimicrobiana y antiparasitaria. Se considera que la planta Bixa orellana puede ser una fuente potencial para la obtención de nuevos agentes antimicrobianos, se ha considerado que la 9-cis-norbixina y todotrans-norbixina son los responsables de sus propiedades antimicrobianas, los resultados de varios estudios demostraron que el achiote tiene efectos inhibidores sobre varios patógenos (Tabla 2). También se detectó actividad ante la Neisseria gonorrhoeae, Trichomonas vaginalis, $T$. faecalis, y Leishmania, siendo superior contra gérmenes gram positivos, aunque también se demostró en algunos gram negativos (Pérez y Martínez, 2010).

Tabla 2. Acción inhibidora de achiote sobre algunos patógenos

\begin{tabular}{lc}
\hline \multicolumn{1}{c}{ Patógeno } & Concentración (V/V) \\
\hline Bacillus cereus & $0.08 \%$ \\
Clostridium perfingens & $0.31 \%$ \\
Staphylococcus aureus & $0.16 \%$ \\
Streptococcus thermophilus & $0.63 \%$ \\
Lactobacillus casei subsp. Casei & $0.63 \%$ \\
Lactococcus lactis & $0.63 \%$ \\
Paenibacillus polymyxa & $0.63 \%$ \\
Listeria monocytogenes & $1.25 \%$ \\
Enterococcus durans & $2.5 \%$ \\
\hline
\end{tabular}

Fuente: Pérez y Martínez, (2010

- Acción hipoglicemiante. Estudios realizados en animales, permitieron demostrar la influencia del achiote en episodios hipoglicémicos mediados por un incremento de la concentración de insulina en el plasma, así como, un 
incremento en la unión de la insulina a sus receptores por un aumento de la afinidad entre ambos (Lourido y Martínez, 2010).

- Acción hipolipemiante, vasorelajante y hepatoprotectrora. En relación con las propiedades hipocolesterolémicas se realizó un estudio es perros, ratones y conejos, que demostró la eficacia (reducción de forma significativa concentraciones de colesterol) del extracto de achiote obtenido de la maceración de las semillas, en las pruebas clínicas, los resultados se confirmaron a los 10 días de la ingesta, por lo tanto los pigmentos pueden acelerar el metabolismo de las grasas, al ser responsables de la reducción de los triglicéridos y colesterol del que, por algún mecanismo aun no descrito, solo reduce la fracción mala (Toledo de Oliveira et al., 2004). Efectos hipotensores también han sido reportados para los extractos de achiote (Vonthron et al., 2003).

- Acción antimutagénica y antigenotóxica. Un estudio evaluó el potencial antimutagénico de la norbixina contra mutágenos oxidantes en Salmonella typhimurium y detectó una inhibición máxima de un $87 \%$ contra este cambio inducido por el peróxido de hidrógeno, sin embargo, se considera que, aunque los datos indican que la norbixina protege frente al daño oxidativo, puede tener efecto pro-oxidante frente al ADN bajo determinadas condiciones. Resultados muy favorables, se observaron en la prevención del daño al ADN en cultivos de linfocitos humanos con el empleo de un extracto de bixina. Por otra parte, los resultados de un estudio realizado en ratas Wistar sugiere un posible efecto quimiopreventivo del achiote a través de la modulación de la proliferación de las células criptales, pero no en la etapa inicial de la carcinogénesis de colon. Otros estudios describen las potencialidades del achiote para prevenir las mutaciones que conducen al cáncer junto a propóleos y algunos hongos comestibles. También se han explicado las potencialidades antimutagénicas del achiote sobre la base de ensayos que demostraron la importante acción de la bixina en la disminución de la peroxidación lipídica (proceso inducido por la formación de radicales libre) y la 
proliferación de células tumorales. Gran parte de estos estudios clasifican a la Bixa como un suplemento nutricional antitumoral (Lourido y Martínez, 2010).

- Acción como antiagregante plaquetario. Conjuntamente con otras 16 plantas medicinales, Bixa orellana fue investigada para demostrar su capacidad para prevenir la agregación plaquetaria. $B$. orellana inhibió la agregación inducida por la trombina de plaquetas humanas lavadas (Kroes y Verger, 2004).

- Acción inmunomoduladora. La bixina se encuentra entre otros colorantes solubles en agua, aumenta la producción de $\lg \mathrm{M}$ a concentraciones tan bajas como $1 \mathrm{mmol} / \mathrm{L}$. este colorante puede regular la producción de inmunoglobulinas (Kroes y Verger, 2004).

- Acción como revelador de placa dentobacteriana. Un estudio realizado en perros Beagle demostró las potencialidades del achiote para la tinción de la placa dentobacteriana, con resultados muy similares a la tinción lograda con el revelador convencional (PLACDENT), cuya formulación es totalmente sintética, el revelador elaborado a base de Bixa orellana (BIXADENT), en cambio es una formulación natural (Gutiérrez, 2005).

\section{DIVERSIDAD DE MATERIALES DEL ACHIOTE}

El achiote es una especie alógama con un alto porcentaje de polinización cruzada que se acentúa cuando se propaga por semilla y en la descendencia se expresa en variedad de formas, tamaño y coloración de las cápsulas y plantas. Es un cultivo con una amplia variabilidad genética (Pérez et al., 2003), éste es del orden del $56.18 \%$ entre arboles y el $14.5 \%$ entre panículas en un mismo árbol, razón por la cual se ha hecho difícil poder encontrar plantas con características fenotípicas que definan un tipo de planta, aunque hay caracteres que se mantienen en este cultivo, que están relacionados con el color de la flor que puede ser blanco o rosado; la cápsula dependiendo del tipo puede ser redonda, acorazonada, lancetada, oblonga, con setas o sin setas (Gutiérrez, 2005). 
Las variedades de achiote se diferencian por las características fenotípicas que las plantas expresan al crecer; entre ellas existen diferentes tipos que las definen e identifican, tales como: flores blancas y cápsulas verdes, flores rosadas y cápsulas con diferentes tonos rojizos. El color de la corteza de los tallos se presenta en gris, rojo, amarillo y verde, sus cápsulas son verdes, rojas, naranjas, café y rojo, con semillas color rojo naranja. De manera similar, la época de floración y cosecha es otra característica que se usa para diferenciar los tipos que existen, es así que la tolerancia natural al mildu polvoriento las clasifica como susceptible o tolerante, es de anotar que también se pueden agrupar en dos tipos de plantas: dehiscentes 0 indehiscentes (Bonilla, 2009).

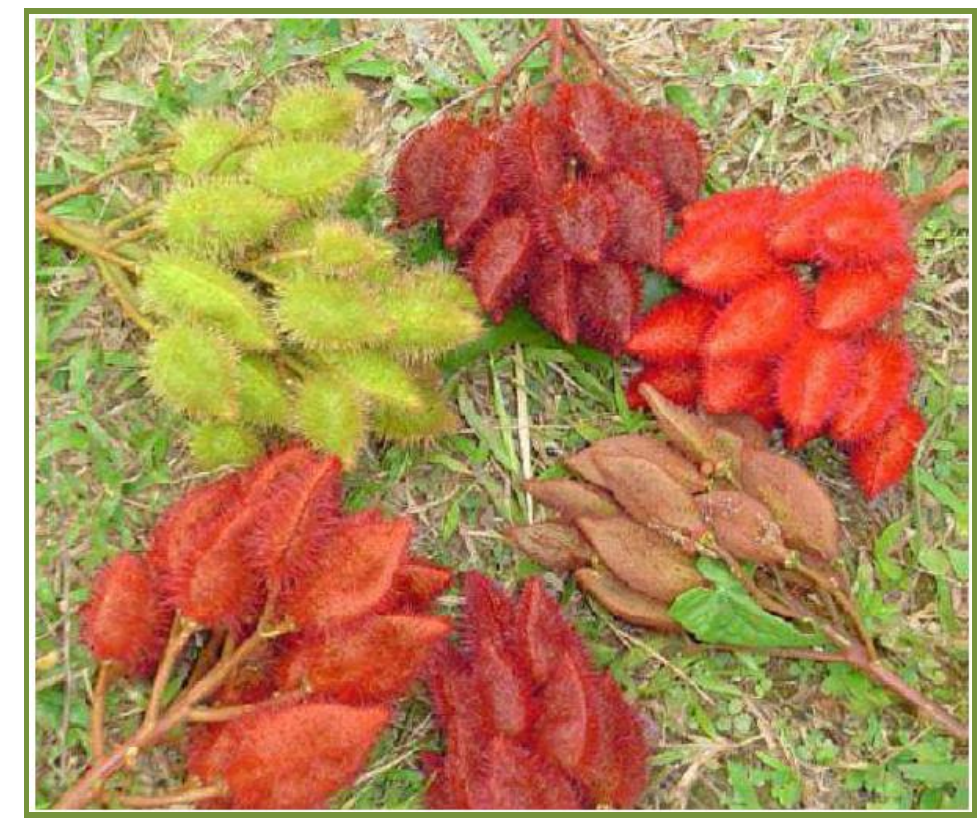

Figura 2. Cápsulas de achiote de diferentes colores. Fuente: Bonilla, 2009.

\section{LA ESPECIE Bixa orellana EN COLOMBIA}

En la actualidad el achiote presenta una alta demanda por parte de los países desarrollados, por sus diversos usos en la industria alimenticia, textil como tinte de velas y sedas, y en la farmacéutica (Cabrera y Piza, 2010). En este sentido, el achiote Bixa orellana es una alternativa productiva en Colombia, debido a que es una especie nativa la cual esta reportada en diferentes regiones del país, en 19 de 
sus 32 departamentos, según los reportes de las colecciones del herbario nacional colombiano, del Instituto de Ciencias Naturales de la Universidad Nacional de Colombia, las cuales datan desde el año 1938, teniendo en el departamento del Chocó el mayor número de colectas (11). Para el caso del departamento del Meta, tiene el reporte más antiguo y registra 5 colectas.

\section{EL ACHIOTE Bixa orellana COMO SISTEMA DE PRODUCCIÓN}

Es una especie de fácil adaptación, se desarrolla muy bien en suelos pobres y ácidos, las plántulas crecen rápidamente, a los 4 meses alcanzan una talla entre 15 y $20 \mathrm{~cm}$, en estado adulto, los árboles son de lento crecimiento. En condiciones naturales los árboles pueden llegar a vivir hasta 50 años y solo 30 en plantaciones, la producción de semillas empieza a los dos años si la plantación ha sido debidamente cuidada, pero su producción normal comienza a los 4 años. Un pequeño árbol de Bixa puede producir de 4,5 a $5 \mathrm{~kg}$ de frutos (Bonilla, 2009). El rendimiento anual promedio de un árbol adulto de cinco años es de 2,33 kg de semilla, unas 1.111 plantas por hectárea rinden en promedio $2.597 \mathrm{~kg}$ de semilla.

\section{Requerimientos edafoclimáticos}

Las temperaturas ideales para su cultivo se encuentran entre los 20 y $38^{\circ} \mathrm{C}$, la precipitación entre 600 y $2000 \mathrm{~mm}$ al año y está relacionada con la condiciones del suelo, puesto que algunos favorecen el desarrollo radicular siendo menor la exigencia del agua, si el suelo tiene una estación seca de 4 a 5 meses, se favorece el desarrollo de la raíz y el achiote crece con una menor cantidad de lluvia, lluvias intercaladas con una estación seca de 4 a 5 meses, y una precipitación total de 1.700 a $2.000 \mathrm{~mm}$ anuales se consideran como aceptables. La humedad relativa más adecuada para el cultivo del achiote varía entre 60 y $80 \%$, cuando es superior se propicia para el desarrollo de hongos, especialmente el Mildu Polvoriento y la Antracnosis, favoreciendo la presencia excesiva de insectos dañinos. Por otro lado, presenta un amplio rango de adaptación entre los 30 y los $1.200 \mathrm{msnm}$, sin embargo, se comporta mejor entre los 300 y $600 \mathrm{msnm}$; la producción de semillas decae cuando se establece esta planta por encima de 
los 800 metros de altitud, el achiote crece pero se desarrolla con limitaciones en la producción y tiene una mayor incidencia de enfermedades. Esta planta tiene tolerancia a vientos fuertes debido su sistema radicular, sin embargo, áreas con vientos huracanados permanentes son perjudiciales para las flores y hojas, causando perdida y daño en las ramas, adicionalmente es necesario tener en cuenta que el achiote requiere de una buena luminosidad, distribuida uniformemente sobre su copa para fructificar con mayor uniformidad. Los índices más elevados de horas de sol, corresponden al periodo de floración y fructificación, que coincide con la estación seca; la cantidad de luz necesaria es 10 horas/día. El achiote se adapta bien a las diferentes clases de suelos siempre que tengan buen drenaje, una profundidad mayor de $0.9 \mathrm{~m}$, textura franco arenosa, con pendientes menores del $5 \%$, siendo la profundidad ideal $3 \mathrm{~m}$, no puede resistir suelos mal drenados, y con alto contenido de arcilla o que posean una capa endurecida. Las mejores plantaciones están normalmente en suelos con un pH de 5 a 7.5 , aunque el achiote soporta pH entre 4.3 y 8.7 (Bonilla, 2009).

\section{Propagación}

Se hace comúnmente de manera sexual, que es la más utilizada, siendo necesario hacer una buena selección de la semilla para garantizar una buena plantación y establecer un vivero. La desventaja de este método radica en que, debido a la polinización cruzada del achiote, se produce una gran variabilidad de individuos y no se puede mantener la uniformidad y la calidad de los frutos, aunque permite tener plantas a corto tiempo para establecer plantaciones comerciales (Avalos, 2013).

El otro método es la forma asexual, que se hace mediante acodos, injertos, estacas o esquejes, cortes de raíz y biotecnología (clones), esto con el fin de mantener la pureza genética completa y conservar las características deseables como el contenido de bixina, el número de semillas por cápsula, el número de cápsulas por racimo, el número de racimos por árbol y tolerancia a mildu polvoriento, se utilizan varias formas de multiplicación (Avalos, 2013). 
- Acodo aéreo: se hace dos meses antes de la siembra definitiva para lo cual se seleccionan los árboles y las ramas donde se colocaran los acodos, efectuando el anillado en las ramas seleccionadas, cortando la corteza en forma de anillo de $2 \mathrm{~cm}$ de ancho y se quita la corteza dejando al descubierto el tejido leñosos, se cubre totalmente con musgo y arena o con una mezcla de arena y materia orgánica, se coloca encima un plástico amarrándolo en ambos extremos y se le hacen agujeros, para poder regarlos y mantener la humedad de la mezcla hasta que la rama haya enraizado; esto ocurre aproximadamente a los 2 meses, sino se mantiene la humedad el acodo se puede perder (Avalos, 2013).

- Acodo al suelo: se seleccionan ramas que estén cercanas al suelo y se procede a efectuar el anillado de la misma manera que se hace para el acodo aéreo, la rama se entierra y se sujeta con unas estacas; esta listo en 2 meses para la siembra definitiva. La desventaja de esta forma de propagación radica en la dificultad de encontrar suficientes ramas para la producción masiva de plantas y hacer plantaciones de gran extensión (Avalos, 2013).

- Injerto: en primer lugar, es necesario obtener un árbol de achiote como patrón propagado por semilla; está listo cuando tiene el grosor de 1 a $1.5 \mathrm{~cm}$. En segundo lugar se necesita una yema de un árbol de achiote seleccionado, este injerto es del tipo parche y consiste en quitar la corteza haciendo un corte en forma de "U" ya sea normal o invertida; la vareta corta yema deberá tener el mismo grosor que el corte en " $U$ ", el injerto se sujeta mediante amarre con cinta plástica; aproximadamente a los veintiséis días se quita el amarre del injerto, luego 10 días más se observa si el injerto está totalmente pegado; si se hubiere pegado se procede a agobiar el patrón para estimular el brote de la yema, y cuando esté aun verde, se elimina la dominancia apical del patrón para dejar libre el injerto; al completar tres meses en los viveros los injertos están listos para la siembra definitiva. También puede utilizarse el injerto por escudete (el mismo que se utiliza en rosas y cítricos), el cual es uno de los más conocidos y requiere solamente una yema para cada injerto. Los arbolitos 
se pueden injertar con yemas provenientes de una selección cuando tienen un grosor de $1 \mathrm{a} 1.5 \mathrm{~cm}$ y la yema se coloca a $0.2 \mathrm{~m}$ del suelo (Avalos, 2013).

- Estacas. El material de madera dura leñosa se coloca en área preparada con arena mezclada con tierra o solo arena, estas áreas deben estar protegidas de la luz directa del sol, colocando sombrío sobre el área permite que la luz directa se reciba por las plántulas en las horas más frescas de la mañana y de la tarde, y no al mediodía. Es necesario mantener la humedad del suelo para garantizar el mayor porcentaje de brote de raíces, puede usarse enzimas para acelerar el brote de las nuevas raíces. Una vez enraizadas las estacas, a los dos meses se siembran en bolsas plásticas negras de vivero medidas 9" x 12" en las que permanecen durante dos meses para después sembrarlas en el terreno definitivo. Existe la misma dificultad que con acodos cuando se quiere hacer una plantación extensiva (Avalos, 2013).

- Cortes y rebrotes de raíz: el achiote puede rebrotar sin dificultad, es una forma de división vegetativa que se hace cortando la raíz estando aun enterrada, lo cual significa hacer uno o vario cortes transversales al grosor de la raíz dejándola enterrada. En el lapso de dos meses se tiene una nueva planta con características similares a la planta madre. Es ideal para hacer clones cuando se tiene poco material para propagar (Avalos, 2013).

\section{Establecimiento de la especie}

Para el establecimiento de la especie Bixa orellana se requiere de una etapa de vivero, la época propicia para su preparación es cuatro meses antes de la siembra en el sitio definitivo al inicio de la época lluviosa, los materiales necesarios son: bolsas negras medidas 9" × 12", tierra negra, materia orgánica, y arena, las tres en proporciones iguales; este sustrato de debe desinfectar, para ello existen varias formas como el uso de fumigantes, para lo cual se recomienda hacer eras con el sustrato, las cuales deben tener $20 \mathrm{~cm}$ de alto y $1 \mathrm{~m}$ de ancho, este sustrato debe permanecer húmedo por mínimo siete días, antes de aplicarle el fumigante, al aplicar el producto se debe regar y posteriormente agregarle Dazomet $^{\circledR}$, en 
proporción de $27 \mathrm{~g} / \mathrm{m}^{2}$, se debe incorporar inmediatamente al sustrato y cubrir con plástico transparente por quince días, posteriormente se retira el plástico y se pica este sustrato y se deja al aire libre por siete días, después de esto estará listo para llenar las bolsas.

Por cada dos metros cuadrados de era se aplica la mezcla de fungicidas de Derosal $^{\circledR}+$ Previsur $^{\circledR}$, en dosis de siete $\mathrm{ml}$ de cada producto por galón de agua, se cubre con plástico durante un día, después de esto el sustrato queda listo para llenar las bolsas. Se incorpora dos onzas de hidróxido de calcio (alcalinizante) por metro cuadrado a una era de $20 \mathrm{~cm}$ de alto por un metro de ancho y el largo necesario para colocar bolsas y plantas que se van a producir. Es importante que en el vivero se evite el uso de bolsas muy pequeñas, la sombra, el exceso de humedad y no es recomendable sembrar en forma tardía si no existe fuente de agua para el riego en la propiedad, las eras muy anchas y muy largas dificultan las labores de mantenimiento (Avalos, 2013).

Antes de sembrar la semilla se coloca en una solución fungicida, con el fin de evitar enfermedades por hongos, se seca a temperatura ambiente en la sombra, posteriormente, se siembra a un centímetro de profundidad, manteniendo la humedad constante en la bolsa mediante el riego diario, sin provocar excesos de humedad ya que causa pudrición de la semilla. Entre los días 5 a 21 las semillas germinan, se dejan una a dos semanas y se seleccionan las más vigorosas (Devia y Saldarriaga, 2003). Posterior a la germinación es necesario mantener la humedad sin llegar a excesos, el tiempo de permanencia de la plántula en el vivero es cuatro meses, en los cuales se debe hacer inspecciones para evitar la presencia de enfermedades y evitar el ataque de insectos plaga, además de mantener libre el lugar de malezas (Bonilla, 2009).

\section{Preparación del suelo}

Los suelos planos y semiplanos mecanizables se recomienda prepararlos con base en la condición del mismo con implementos agrícolas, en áreas onduladas y laderas, es mejor realizar el ahoyado para ofrecer condiciones favorables en el 
crecimiento de la planta, utilizando herramientas manuales. Los hoyos para siembra de achiote tienen dimensiones de $0.5 \mathrm{~m} \times 0.5 \mathrm{~m} \times 0.5 \mathrm{~m}$, y deben ser cubiertos con materia orgánica por lo menos dos meses antes de la siembra definitiva (Bonilla, 2009).

\section{Siembra}

La siembra por trasplante es un sistema alternativo que permite inicialmente hacerlo en bandeja y luego sembrarlas en las bolsas para brindar mayores cuidados a la planta al inicio del crecimiento en vivero. El mantenimiento y la supervisión permiten llevar al campo plantas vigorosas, de buena calidad y que estarán litas para ser trasplantadas de 3 a 4 meses de edad (Bonilla, 2009). Los sistemas de producción agrícola moderna de plantas permanentes están orientados a las altas densidades de siembra con el manejo de plantas bajas, con el objeto de que se haga más fácil recolectar la cosecha, recomienda sembrar a distancia de 3 × $3 \mathrm{~m}$ para lograr una densidad de 1.111 árboles por hectárea (Bonilla, 2009). El achiote se puede asociar preferiblemente con cultivos de ciclo corto y fácil manejo, por ejemplo: maíz, ají, frijol, pepino, hortalizas, plantas aromáticas y con variedades de árboles frutales o incluso árboles forestales.

\section{Manejo del cultivo}

Existe poca información sobre los requerimientos del achiote, en Venezuela se han obtenido cosechas precoces y abundantes con el plan de manejo de fertilización (Tabla 3), previo a un análisis de suelo, y control manual o químico de las arvenses para evitar la competencia por luz, nutrientes, agua y espacio, de éstas con el achiote, iniciando la siembra a principios del periodo lluvioso (Bonilla, 2009). El achiote es relativamente resistente a condiciones de sequía, pero se debe garantizar una lamina de agua suficiente que humedezca la zona de las raíces; en sitios de alta precipitación no es necesario el riego suplementario; en lugares muy soleados, el riego por surcos una vez a la semana después de que el cultivo se ha establecido es suficiente. La lámina de agua a aplicar debe ser 
suficiente para que humedezca completamente la profundidad radical del cultivo (Sahaza, 2001).

Tabla 3. Plan de fertilización en el cultivo de achiote

\begin{tabular}{|c|c|c|c|}
\hline $\begin{array}{c}\text { Etapa } \\
\text { (meses) }\end{array}$ & $\begin{array}{c}N \\
\text { (g/planta) }\end{array}$ & $\begin{array}{c}\mathrm{P}_{2} \mathrm{O}_{5} \\
\text { (g/planta) }\end{array}$ & $\begin{array}{c}\mathrm{K}_{2} \\
\text { (g/planta) }\end{array}$ \\
\hline \multicolumn{4}{|c|}{ Primer año de siembra (inicio de lluvias) } \\
\hline Siembra & 15 & 30 & 15 \\
\hline 3 & 40 & 0 & 0 \\
\hline 6 & 15 & 30 & 15 \\
\hline 12 & 50 & 0 & 0 \\
\hline \multicolumn{4}{|c|}{ Segundo año } \\
\hline A floración & 30 & 60 & 30 \\
\hline 4 & 60 & 0 & 0 \\
\hline 8 & 30 & 60 & 30 \\
\hline 12 & 60 & 0 & 0 \\
\hline \multicolumn{4}{|c|}{ Tercer año } \\
\hline A floración & 60 & 120 & 60 \\
\hline 4 & 120 & 0 & 0 \\
\hline 8 & 60 & 120 & 60 \\
\hline 12 & 120 & 0 & 0 \\
\hline
\end{tabular}

Fuente: Sahaza, 2001

De acuerdo a un trabajo de experimentación realizado por la Universidad del Valle, se sugiere utilizar el riego por goteo para optimizar el uso del agua (Tabla 4), se hace teniendo en cuenta condiciones climáticas de esta zona del país, teniendo en cuenta que el drenaje depende de la topografía, en algunos casos no son necesarios los drenajes porque el terreno lo hace de manera natural, que para el desarrollo de las plantas, el suelo tenga el perfil y la humedad apropiada, pues las raíces de Bixa orellana no soporta el encharcamiento (Avalos, 2013).

Con las podas lo que se busca es estimular a la planta para producir la mayor cantidad de brotes terminales, donde se forma la flor y el fruto, esta práctica mantiene la plantación rejuvenecida y aumenta la producción, la densidad de población por unidad de superficie, facilitando la cosecha en menor tiempo y costo. Todo tipo de poda debe de efectuarse inmediatamente después de la 
cosecha, existen cuatro tipos: 1) la de formación, generalmente se logra en el primer año, y consiste en dejar un solo tallo y las ramas principales a $30 \mathrm{~cm}$ de largo desde el tallo principal; 2) la de mantenimiento, se hace para preservación de la poda anterior, eliminando los brotes basales, y se realiza en los tres primeros años; 3) la anual de limpieza, a partir del segundo año en adelante se debe eliminar brotes vegetativos en la base de la planta y ramas secas; y 4) la poda de rejuvenecimiento o recepa se realiza a los 4 o 5 años a fin de evitar que la planta crezca demasiado alta y la cosecha pueda colectarse fácilmente, consiste en cortar el árbol severamente, dejando los brotes nuevos (Sahaza, 2001).

Tabla 4. Recomendaciones de riego para el cultivo de achiote

\begin{tabular}{lccc}
\hline \multicolumn{1}{c}{ Fase } & $\begin{array}{c}\text { Frecuencia } \\
\text { (Horas) }\end{array}$ & Litros por planta & $\begin{array}{c}\text { Duración } \\
\text { (Horas) }\end{array}$ \\
\hline Prendimiento & 36 & 10,7 & 1 \\
Desarrollo & 36 & 21 & 2 \\
Productivo & 36 & 32 & 3 \\
\hline
\end{tabular}

Fuente: Avalos, (2013)

\section{Plagas}

Durante el desarrollo del cultivo del achiote se pueden presentar problemas con varias plagas, la mayoría de ellos no se consideran de importancia económica, entre los que se encuentran insectos chupadores, chinche patas de hoja (Leptoglossus zonatus); masticadores, el zompopo (Atta mexicana); araña roja (Tetranychus sp.); trips de banda roja (Selenothrips rubricinctus Girard) y barrenadores como el perforador de la cápsula (Milgithea melanoleuca). Una de las prácticas para su control es realizar eliminación de residuos y malezas, además de la utilización de parasitoides, depredadores y patógenos (enemigos naturales), cuando el ataque es severo, se debe implementar un control químico con la aplicación de insecticidas al cultivo (Bonilla, 2009).

- Chinche patas de hoja (Leptoglosus zonatus): ocasiona pérdidas económicas en la producción de achiote, cuando ataca la cápsula en desarrollo, 
ocasionando daño secundario por hongos en las semillas, el cual se observa en la cápsula cuando el chinche ya lo ha provocado con su estilete, dando la impresión de que la cápsula se está madurando, también se forma una mancha necrótica en la cápsula y las semillas se cubren de un micelio de un hongo secundario. El chinche tiene hábitos gregarios en su estado de ninfa y no vuela, por lo que en esta etapa puede ser fácilmente controlado (Bonilla, 2009).

- El zompopo (Atta mexicana): destruye el follaje tierno, su ataque intenso puede defoliar completamente la planta y no dejarla progresar, ya sea que la planta se encuentre en su etapa juvenil o adulta; esta plaga en vivero causa muchos destrozos por lo que es necesario estar pendiente del primer ataque, para su control (Bonilla, 2009).

- Araña roja (Tetranychus sp.): estos ácaros se alimentan succionando la savia de las plantas, produciendo defoliación, en la época seca se presentan poblaciones mayores que agravan el daño, al secar las hojas se decoloran y caen (Bonilla, 2009).

- Trips de banda roja (Selenothrips rubricinctus Girard): se alimenta de la savia de las plantas, raspando el envés de las hojas dándole apariencia de requemo, ataques severos resultan en defoliación afectando la floración, la polinización y fructificación (Bonilla, 2009).

- El perforador de la cápsula (Milgithea melanoleuca): la hembra de la mariposa coloca los huevecillos sobre las cápsulas, la humedad y la temperatura son propicios para que eclosionen los huevecillos, al eclosionar salen las larvas que perforan las cápsulas y se alimentan de las semillas (Bonilla, 2009).

- Comején (Hetotermes convexinotatus Zinder): penetra en el árbol por cualquier cortadura en las raíces y perfora todo el xilema del árbol hasta que lo seca completamente, siendo el mejor control, no sembrar debajo de los 300 msnm donde exista el problema del comején. Una práctica recomendable es 
no dejar dentro de la plantación ramas que se hayan podado, evitando el daño de raíces cuando se limpie las malezas del cultivo (Bonilla, 2009).

\section{Enfermedades}

Para prevenir y controlar la presencia de las enfermedades en el cultivo se recomienda utilizar semilla sana y limpia, mantenerlo libre de arvenses, evitando el encharcamiento, desinfectar las herramientas o equipo de poda, retirar del cultivo plantas enfermas o las partes de algunas de ellas que estén afectadas. Las principales enfermedades reportadas en el achiote son:

- Oídium, ceniza, moho blanco, mildu polvoriento (Oidium Bixae): se presenta en forma de manchas blancas, causadas por un micelio pulvurulento blanquecino sobre las hojas, ramos y frutos jóvenes, provocando una defoliación, principalmente en plantas jóvenes, sobre todo en épocas lluviosas o cuando el punto de rocío es muy alto (OTCA, 1999).

- Muerte descendente o antracnocis (Colletotrichum gloeosporioides): los síntomas aparecen en las ramas jóvenes, las cuales pierden su follaje y se secan., avanzando la necrosis a las ramas secundarias y a las primarias, cuando la infección es severa provoca la muerte de la planta, la cual va muriéndose de arriba hacia abajo (de ahí proviene el nombre de la enfermedad) (Arce, 1999).

- Podredumbre de las cápsulas o antracnosis de las cápsulas (Colletotrichum gloeosporioides): este hongo ataca únicamente las cápsulas de las semillas en estado de desarrollo, en una etapa muy temprana, no se desarrollan semillas dentro de la cápsula, o las pocas q lo hacen están deformadas. Los síntomas visibles en la cápsula son la aparición de manchas negras pequeñas y circulares, que con el paso del tiempo se unen formando una mancha grande. El hongo puede penetrar el interior de las cápsulas y cubrir las semillas con un micelio blanco, destruyéndolas por completo (Arce, 1999). 
- Escoba de bruja (Crinipellis pernicosa): este hongo causa proliferaciones (hipertrofia) anormales de los brotes laterales, así como un hinchamiento de las ramas, inicialmente estos brotes tienen una coloración verde, pero progresivamente se van secando, hasta adquirir un color marrón oscuro (OTCA, 1999).

- Pudrición de los frutos (Fusarium sp.): este hongo causa manchas de forma y tamaño variables, los cuales se necrosan, las semillas se pudren y son cubiertas con un micelio algodonoso, el cual se observa a través de rajaduras que se presentan en los frutos muy atacados (OTCA, 1999).

- Mancha parda de las hojas (Cercosporae bixae): esta enfermedad se presenta tanto en plantas jóvenes y adultas, hay presencia en las hojas de manchas irregulares de color marrón a ceniza, rodeadas por un borde púrpura y un halo amarillento u oscuro, posteriormente provoca perforaciones en la lámina de las hojas y luego una defoliación prematura. Aun cuando el hongo principal que se ha aislado es Cercospora bixae otros hongos como Stiibum sp., Phyllosticta sp., Phoma sp., pueden causar los mismos síntomas y daños (Arce, 1999).

- Podredumbre de los semilleros "Damping off": es causada por hongos Rhizoctonia, que viven en el suelo y atacan las raíces, cuello y tallo de las plántulas recién emergidas, siendo el primer síntoma es el marchitamiento, causando posteriormente inclinación y caída de la planta (Arce, 1999).

- Pudrición negra de las raíces: ataca las raíces de las plantas jóvenes y adultas, es causada por el hongo Rosellinia sp. Los primeros síntomas que se observan son el amarillamiento y caída de las hojas, finalmente la planta muere; en el sistema radicular de la planta se puede observar el micelio del hongo y las lesiones (OTCA, 1999).

\section{POSTCOSECHA}

Recolección. En condiciones del trópico la primera floración aparece a los 9 meses, presentándose la primera cosecha a los 11,5 meses, en regiones muy 
secas la primera cosecha es a los 18 meses, esta es de alto rendimiento (hasta $2.500 \mathrm{~kg} / \mathrm{ha}$ ) se presenta producción durante todo el año, aunque puede observase dos picos de alta producción cada seis meses.

Un método para determinar la madurez de cosecha es la firmeza de la cápsula (Figura 3), al estar dura al contacto estará lista, también es útil como indicador de madurez el momento en el que la cápsula empieza a perder la intensidad de su color (Bonilla, 2009), iniciando a tornarse café. La mayoría de variedades de Bixa orellana presentan frutos dehiscentes y esto también puede ser un indicador de madurez, aunque se debe evitar la dehiscencia o apertura de la cápsula para que la degradación por luz no dañe o altere las propiedades de los colorantes como la bixina y la norbixina en las semillas. No es recomendable realizar la cosecha de frutos inmaduros, ya que la concentración de bixina y norbixna puede estar aún bajas, y en el manejo postcosecha los frutos pueden presentar fermentación, debido a los mucílagos que cubren las semillas frescas.

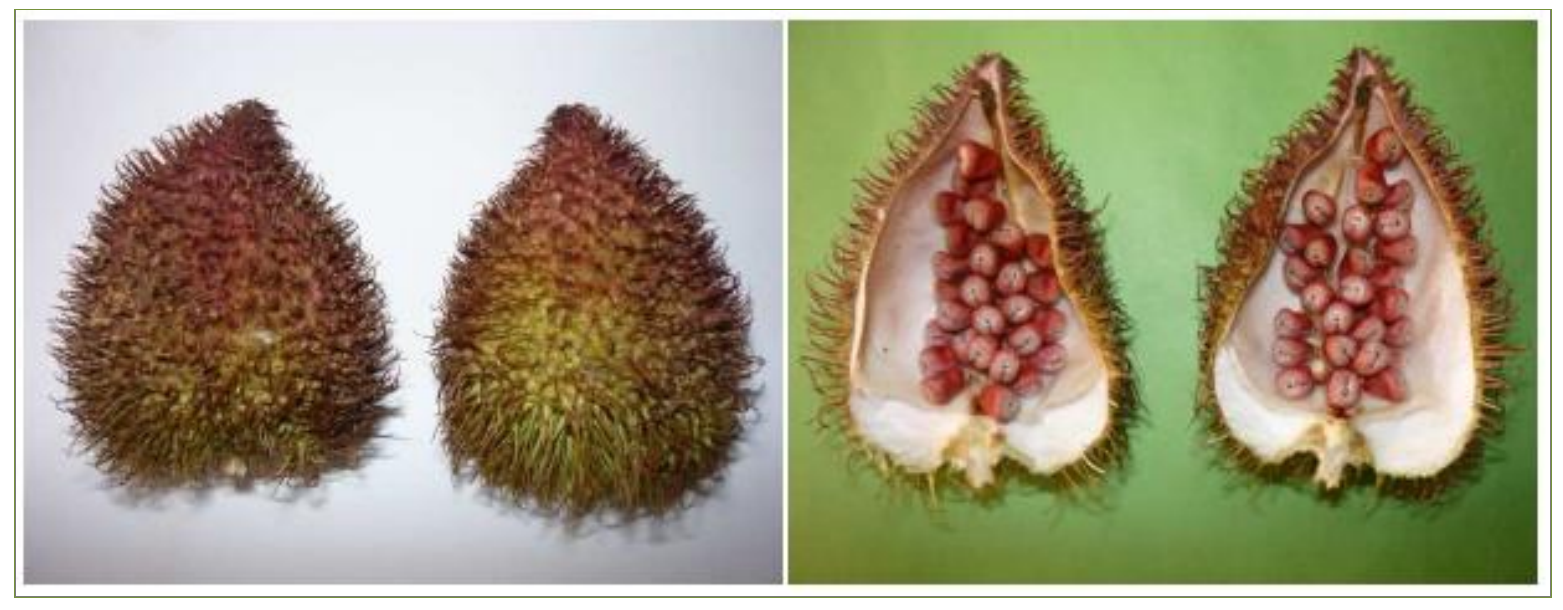

Figura 3. Cápsulas y semillas de achiote Bixa orellana.

Las cápsulas terminales son siempre las últimas en madurar y cuando están maduras, las inferiores pueden estar secas, los racimos deben ser cortados con tijeras bien afiladas justo por encima del primer nudo del manojo, si la cosecha se efectúa tarde, las cápsulas tendrán semillas germinadas o con hongos, lo cual afecta la calidad del producto (FAO, 2006). 
Separación, pesado y limpieza. Las cápsulas se deben dejar secar hasta que las semillas se desprendan fácilmente de sus paredes, lo cual toma aproximadamente unas 50 a 60 horas de insolación (FAO, 2006), no se debe exponer las semillas a temperaturas mayores de $60^{\circ} \mathrm{C}$, ni a excesos de luz, tampoco se recomienda extraer la semilla de la cápsula si ésta se encuentra húmeda. El porcentaje de humedad de la semilla debe estar entre 8 y $12 \%$. Las semillas se pesan separadas de las cápsulas y luego se realiza una limpieza, si están muy sucias se debe lavar con agua, de lo contrario se podría hacer una limpieza con un paño húmedo (FAO, 2006).

Empaque y almacenamiento. Una vez seca la semilla con 8 a $12 \%$ de humedad, se empaca en sacos de fique o de material biodegradable, el uso de bolsas oscuras conlleva a una mejor estabilidad del colorante durante el almacenamiento, lo cual no influye en la concentración de bixina de la semilla, se puede almacenar hasta un año y medio, sin que se alteren las sustancias colorantes. Las cápsulas o las semillas separadas se deben almacenar a granel en silos o contenedores herméticos, protegidos de la luz (FAO, 2006).

\section{PRODUCTOS Y SUBPRODUCTOS DE LA PLANTA ACHIOTE}

De las ramas se obtiene una goma que es similar a la goma arábiga, una resina de algunas especies de acacias que disuelta en agua se puede usar como adhesivo de algunos esmaltes cerámicos, la madera del tronco se utiliza para la construcción de muebles, en construcciones rurales, como fuente de energía y en la elaboración de implementos o mangos para herramientas agrícolas, su corteza contiene una fibra para cordelería, de la porción carnosa (arilo rojo-naranja) que envuelve a la semilla se extrae un colorante apreciado industrialmente para dar olor a productos alimenticios (queso, mantequilla), aceites, ceras, barnices, pinturas, cosméticos y fibras textiles (de seda y algodón) (Arce, 1999). Por otro lado, el aceite de las semillas es emoliente y con un contenido alto en carotenoides que le provee propiedades antioxidantes, se ha utilizado en productos para el cuidado del cuerpo como cremas, lociones y champú. La semilla 
molida se utiliza para el tratamiento de enfermedades como sarampión, viruela, enfermedades del riñón, disentería y febrífugo, como astringente y ligero (Irobi, et al., 1996). El polvo de las semillas es considerado como un afrodisíaco, aparte de sus propiedades estimulantes y digestivas, siendo sus semillas y frutos un antídoto eficaz contra envenenamiento por Jatropha curcas (dolores abdominales, diarrea, vómito, irritación de garganta, gastroenteritis) y Manihotes culenta (manifestaciones disneicas). De la raíz se obtiene un té hecho con pequeños vástagos y hojas que es usado como antidisentérico, afrodisíaco, astringente y para tratar infecciones de la piel, fiebres y hepatitis, los extractos en alcohol etílico de frutos y hojas han mostrado tener actividad in vitro contra Escherichia coli y Staphylococcus aureus (Irobi, et al., 1996).

\section{MERCADO DEL ACHIOTE Bixa Orellana $L$.}

De 2005 a 2009, el mercado mundial de colorantes naturales aumentó casi un $35 \%$, situación que continuará si se tiene en cuenta que en el procesamiento de alimentos representa alrededor del $67 \%$, seguido por las bebidas sin alcohol con un $28 \%$ y las bebidas alcohólicas con un $5 \%$. Entre los demandantes de estas materias primas naturales, están Europa con el 36\% del mercado mundial de colorantes, seguido por Estados Unidos (28\%), Japón (10\%), China (8\%) y el $18 \%$ restante, corresponde a países con economías desarrolladas como Canadá y Australia, y a mercados emergentes como India y Brasil (López et al., 2009).

De acuerdo a información de la cadena productiva de plantas aromáticas, medicinales, condimentarías y afines, entre el 2003 y 2007 se destacan como los principales importadores a nivel mundial de colorantes, Italia, Reino Unido, Alemania y Francia, Japón también tiene una demanda importante; en el continente Americano Estados Unidos es el mayor importador a nivel mundial, México, Guatemala, Colombia y Brasil hacen parte de esos importadores de colorantes en orden de importancia de acuerdo a la Grafica 1 (López et al., 2009).

Sin embargo, en información de Proexport 2011, el país con mayor participación en las importaciones mundiales de achiote en polvo sigue siendo Estados Unidos 
con un $49.35 \%$, este país muestra un crecimiento en las importaciones de este producto entre el 2007 y 2009 del $82.33 \%$; el segundo lugar lo ocupa Japón con un $13.3 \%$. En Latinoamérica, México es el país con mayor participación en las importaciones con un $1.56 \%$, seguido de Brasil con un $1.17 \%$, Ecuador $0.56 \%$ y Perú con $0.063 \%$. En las exportaciones de colorantes naturales de 2003 a 2007 China es el país con mayores volúmenes de colorantes naturales exportados; en la Unión Europea España, Alemania, Francia e Italia se destacan; en el continente Americano México, Perú, Brasil, Chile, República Dominicana; las exportaciones de Colombia solo se registran en el año 2006 (López et al., 2009) como se muestra en la Gráfica 2.

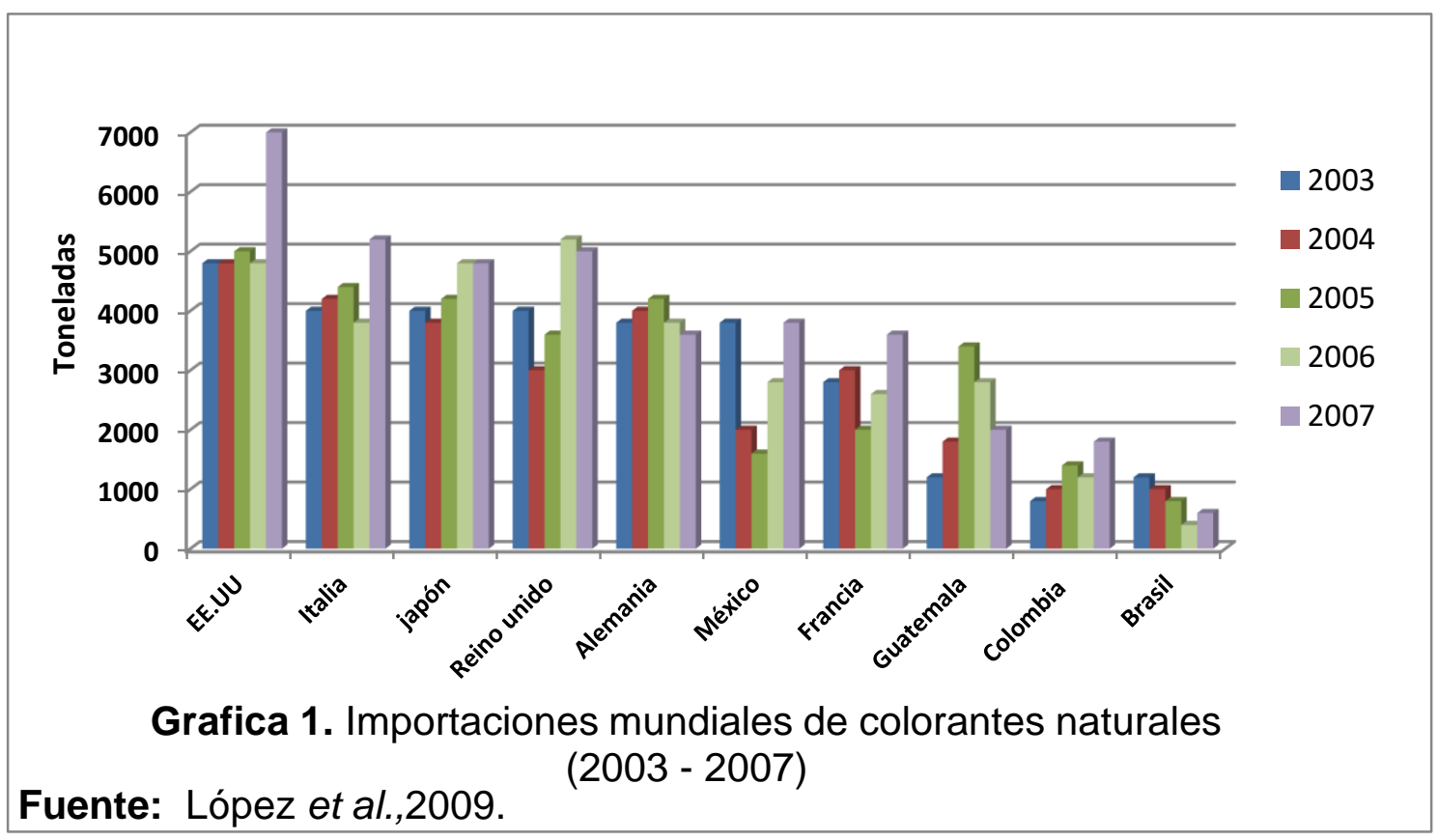

Los proveedores de colorantes naturales en orden de mayor participación en porcentaje son: España (11.4\%), Alemania (9.9\%), Francia (8.7\%), Holanda (8.3\%), Suiza (1.1\%), Australia (0.9\%), Perú (7.1\%), China $(6.0 \%)$, India $(3.9 \%)$, México (1.6\%), Zimbabwe (0.9\%) y Sudáfrica (0.9\%) (López et al., 2009). De acuerdo a información de la FAO, (2006) Perú es el primer productor y exportador mundial de achiote, representa un 35\% de la producción mundial, el otro 65\% proviene de Kenia, Brasil e India. Los principales países a quienes se les vende 
semillas de achiote son los Estados Unidos, Costa Rica, Puerto Rico, Venezuela y Canadá. Los principales países a quienes se les vende el derivado de achiote Bixina/Norbixina son: Estados Unidos (quien está demandando este producto en diferentes concentraciones, siendo la más solicitada Bixina Cristal al 95\%) Japón, Alemania y Holanda.

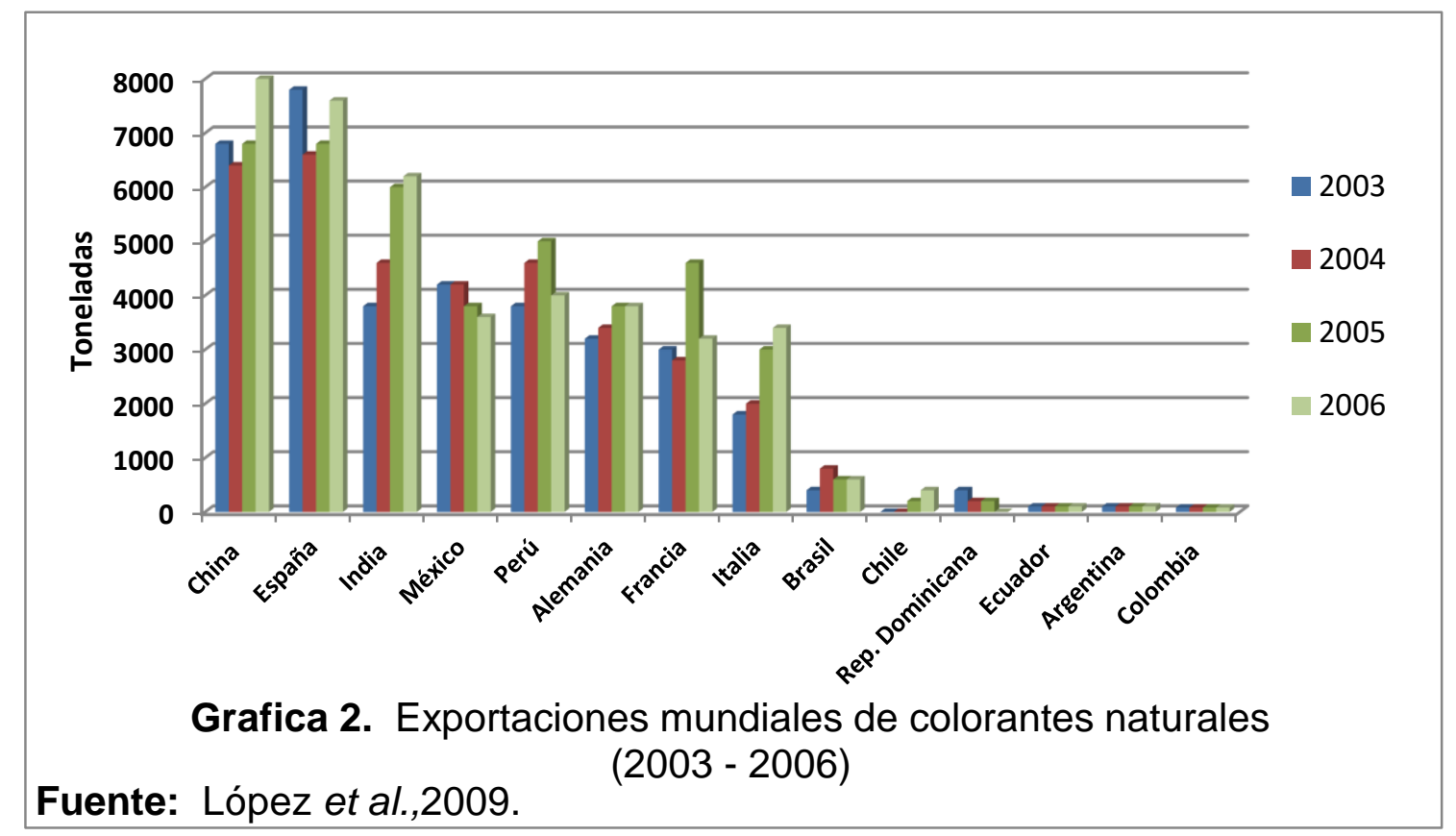

Importaciones y exportaciones de achiote en Colombia

Según los registros de Agronet, Colombia realizó importaciones de Achiote en los años 2007 y 2008, según esta misma estadística, ha realizado exportaciones a Hong Kong y Venezuela en los años 2007 y 2008 respectivamente, con un volumen total que no sobrepasa los $600 \mathrm{~kg}$, y un precio de venta de US\$1.293 (MADR, 2008) (Tabla 5).

Tabla 5. Importaciones de Achiote Bixa Orellana en Colombia

\begin{tabular}{cccc}
\hline Año & Toneladas & Origen & $\begin{array}{c}\text { Precio Implícito } \\
\text { (US } \$ \text { Ton) }\end{array}$ \\
\hline 2007 & 7,0 & Perú & 1.495 \\
2009 & 31,5 & Perú & 194 \\
\hline
\end{tabular}

Fuente: MADR, 2009. 


\section{Producción nacional y regional}

De acuerdo con el Herbario Nacional Colombiano en los reportes de colecciones, se tienen registro de las colectas de Bixa orellana desde 1938, en 19 departamentos del territorio nacional, entre los cuales está el departamento del Meta, con el registro más antiguo y cinco colectas en los municipios de Acacias, Restrepo, Puerto Gaitán y Cabuyaro. Los desarrollos en investigación y procesamiento del achiote encontrados a nivel de Colombia están siendo liderados por la Universidad Nacional sede Palmira, Universidad del Valle en el programa manejo integral de cultivos para el fortalecimiento de la cadena productiva de plantas medicinales, aromáticas y condimentarías (López et al., 2009) y la Universidad Eafit de Medellín, con un trabajo sobre el diseño de un prototipo de planta piloto para obtener el colorante de la semilla del achiote (Bixa Orellana L.) (Devia et al., 2005).

La diversidad de sitios con condiciones agroclimatológicas diferentes, permite concluir que esta planta tiene una amplia capacidad adaptativa, en cuanto a suelos, temperatura, precipitación y altitud, por lo tanto, desarrollar un sistema productivo de achiote en condiciones del departamento es viable. Un posible limitante para el desarrollo de la especie está relacionado con la humedad relativa, puesto que si se encuentra por encima del $80 \%$ favorece el desarrollo de patógenos como Oidium bixae hongo causante de la enfermedad conocida como mildu polvoriento, la cual puede afectar el normal desarrollo de la planta y la producción de frutos de buena calidad, esta condición ambiental es un limitante para el desarrollo de Achiote Bixa Orellana en algunas zonas del departamento del Meta.

La oferta agroclimatológica del Meta, está relacionada con los corredores viales que le permite comunicarse con otras regiones y en particular con la capital del país, esto le da ventajas comparativas en aspectos como costos de transporte y trasformación de las semillas en polvo o extracto de colorante. Por lo anterior, es una prioridad desarrollar conocimiento local sobre el achiote como sistema de 
producción agrícola, en arreglos productivos con cultivos de pancoger, que se constituya en una alternativa social, económica y ambientalmente sostenible para el departamento, en donde una de las mayores limitaciones es la trazabilidad y el conocimiento técnico de los productos.

\section{CONCLUSIONES}

El polvo o extracto de Achiote Bixa orellana, es una alternativa competitiva para el mercado mundial de colorantes naturales, el cual presenta una tendencia creciente en la demanda, con países compradores como Estados Unidos, Japón, Italia, Alemania, Reino Unido y Francia, entre otros.

Las condiciones biofísicas y climáticas del departamento del Meta son adecuadas para el desarrollo del Achiote como sistema productivo, pues la planta se adapta bien en suelos de mediana a baja fertilidad, con texturas franco arenosas o franco arcillosas, con condiciones de buen drenaje, pendientes menores de $5 \%$, precipitaciones que oscilan entre 2000 y $2500 \mathrm{~mm}$ y una temperatura promedio de $24^{\circ} \mathrm{C}$.

\section{REFERENCIAS BIBLIOGRÁFICAS}

1. Arce P. J. El Achiote Bixa Orellana L. cultivo promisorio para el trópico. Ed Universidad Earth. p. 49. 1999. Recuperado 15 de enero 2011. Disponible En: http://usi.earth.ac.cr/glas/sp/90019483.pdf

2. Antunes L, Pascoal L, Bianchi L, Dias F. Evaluation of the clastogenicity and anticlastogenicity of the carotenoid bixin in human lymphocyte cultures. Mutat Res., 585 (1-2): 113-9. 2005.

3. Avalos J. M. Caracterización morfológica de dos variedades de achiote (Bixa orellana) en el municipio de Comalcalco, Tabasco. Tesis como requisito parcial para obtener el grado de Magister en Sistemas Sustentable de Producción en el Trópico. Colegio de Postgraduados. México. p. 50. 2013.

4. Bonilla J. Manual del cultivo de achiote. Proyecto de desarrollo de la cadena de valor y conglomerado agrícola. Nicaragua. Abril 09, 2009. Recuperado 22 de septiembre de 2010.2 Disponible http://www.cenida.una.edu.ni/relectronicos/RENF01B715mc.pdf

5. Cabrera I. M., Piza R. E. Importancia económica del valor agregado de los derivados del achiote en el mercado interno y externo como alternativa de desarrollo comercial y agroindustrial del sector empresarial de Manabí. Período 2005 - 2008. Tesis de grado previo a la obtención del título de 
Economista. Universidad Técnica de Manabí. Portoviejo, Manabí, Ecuador. 129 p. 2010.

6. Centro Nacional de Tecnología Agropecuaria y Forestal. Manual técnico: el cultivo del achiote, Bixa Orellana. El salvador. 19 p. Recuperado 23 de febrero de $2011 . \quad$ Disponible En: http://www.centa.gob.sv/uploads/documentos/manual\%20tecnico\%20del\%20a chiote.pdf

7. Colmenares A. J. Manejo integral de cultivos para el fortalecimiento de la cadena productiva de plántulas medicinales, aromáticas y condimentarías en el centro del Valle del Cauca, orientado a la especie Bixa Orellana. Recuperado 10 de Marzo de 2011. Disponible En: http://www.cultivobixaorellana.com/

8. Comisión Nacional para el Conocimiento y uso de la Biodiversidad, CONABIO. México. Recuperado 2 de marzo de 2011. Disponible En: http://www.conabio.gob.mx/conocimiento/info especies/arboles/doctos/13bixac1m.pdf

9. Comisión Nacional Forestal. CONAFOR. Sistema nacional de información forestal. México. Recuperado 22 de enero de 2010. Disponible En: http://148.223.105.188:2222/gif/snif portal/secciones/usos/usospdf.php?especi eURL=Bixaorellana

10. Corporación para el Desarrollo Sostenible del Área de Manejo Especial la Macarena. CORMACARENA. Plan de gestión ambiental regional 2010 - 2019.

11. Devia J. y Saldarriaga L. Planta piloto para obtener colorante de la semilla del achiote (Bixa orellana). Revista Universidad EAFIT. 39 (131): 10. 2003. Recuperado 13 de diciembre de 2010. Disponible En: http://bdigital.eafit.edu.co/bdigital/HEMEROTECA/HRU038/131Revista.pdf

12. Devia J. E., Constain O. V. R., Brancho D. C. A., Cruz T. D. C., Escobar P. C., Alviar H. S., Villegas M. C., Paredes A. R., Palomino H. C., Londoño S. B. E. et al. Pulverización de colorantes naturales por secado por atomización. Universidad EAFIT. 58 p. 2005.

13. Fonnegra R., Jiménez S. Plantas medicinales aprobadas en Colombia. $2^{\mathrm{a}}$ ed. Editorial Universidad de Antioquia. p 15. 2007. Recuperado 5 de enero de 2011. Disponible En: http://books.google.com.co/books?id=K8el7ZeFpsC\&printsec $=$ frontcover\&dq $=$ medicinales+aprobadas+en+Colombia\&sou rce=bl\&ots $=6 \mathrm{Cr} \times 6 \mathrm{sscy} \& \mathrm{sig}=\mathrm{Lz} 5 \mathrm{hPHm} 7 \mathrm{LH} \quad$ XdhAcjA8Q4J1lv18\&hl=es\&ei=AY I TdOOMMbXgQekm9CiCA\&sa=X\&oi=book result\&ct=result\&resnum=5\&ve

14. FAO, Food and Agriculture Oraganization. El achiote (Bixa orellana). Fichas Técnicas, 2006. Recuperado 14 de marzo de 2011. Disponible En: www.fao.org/inpho/content/docuemntos/vlibrary/ae620s/Pfrescos/ACHIOTE.ht $\mathrm{ml}$

15. Giuliano G., Rosati C., Bramley P. To dye or not to dye: biochemistry of annatto unveiled. Trends in Biotechnology. 21 (12): 513-6. 2003,

16. Gómez E., Hernández J. Plantas medicinales: 2 Métodos de cultivo: Innovación tecnológica. Vol. 16, marzo 2010. Recuperado 24 de septiembre de 2010.

Disponible

En: 
http://innovacion.ciget.lastunas.cu/index.php/innovacion/article/viewFile/178/16 $\underline{8}$

17. González Y., Porta T., Méndez R., Blanco F. Estudio de la irritación dérmica primaria en piel dañada de conejos tras la exposición a un extracto alcohólico de Bixa orellana Lin. Apresentado Em: Conferencia Internacional de Química, 2, Santa Clara, 2003. Disponible En: http://bases.bireme.br/cgibin/wxislind.exe/iah/online/?IsisScript=iah/iah.xis\&src =google\&base $=$ LILACS\&lang=p\&nextAction=Ink\&exprSearch=389046\&indexS earch $=$ ID

18. Gutiérrez M. "Bixadent", nuevo producto natural como revelador de placa dentobacteriana. VI Congreso de la Sociedad Cubana de Bioingeniería. La Habana, $2005 . \quad$ Disponible En: http://www.memsocbio.sld.cu/habana2005/arrepdf/T078.pdf

19. Instituto Nacional de Investigación Agraria, INIA. Accesiones promisorias. Banco de Germoplasma de la SUDIRGEB. Ed Velarde Falconi David y Rios Lobo Llermé. 98 p. 2009.

20. Irobi O. N., Moo-Young M., Anderson W. A. Antimicrobial Activity of Annatto (Bixa orellana) Extract., 34 (2): 87-90. 1996.

21. James A, Duke, Mary Jo Bogenschutz-Godwin, Judi du Cellier, Peggy-Ann Duke CRC. Handbook of Medicinal Spices. Boca Raton: CRC Press; 2003.

22. Júnior A, Asad L, Oliveira E, Kovary K, Asad N, Felzenszwalb I. Antigenotoxic and antimutagenic potential of an annatto pigment (norbixin) against oxidative stress. Genet Mol Res; 4 (1): 94-9. 2005.

23. Kroes R., Verger P. Who Food Additives Series: 52. Annatto Extracts. World Health Organization, Safety evaluation of certain food additives and contaminants. World Health Organ Tech Rep Ser., 922: 1-176. 2004. Erratum in: World Health Organ Tech Rep Ser., 928: 157. 2005. Disponible En: http://www.inchem.org/documents/jecfa/jecmono/v52je03.html

24. López L. F., González D. M., Gómez J. A., Albarracín C. Agenda prospectiva de investigación y desarrollo tecnológico para la cadena productiva de plantas aromáticas, medicinales, condimentarías y afines con énfasis en ingredientes naturales para la industria cosmética en Colombia. Ministerio de Agricultura y Desarrollo Rural. Giro Editores Ltda, Bogotá. 184 p. 2009.

25. Lourido H., Martínez G. La Bixa orellana en el tratamiento de afecciones estomatológicas, un tema aún por estudiar. Revista Cubana de farmacia. 44 (2): 231-244. 2010. Recuperado 04 de marzo de 2011. Disponible En: http://scielo.sld.cu/scielo.php?script=sci arttext\&pid=S0034751520100002000 $12 \& \operatorname{lng}=$ es\&nrm=iso

26. Ministerio de Agricultura y Desarrollo Rural, MADR. Definición de la agenda prospectiva de investigación para la cadena productiva de plantas aromáticas, medicinales y condimentarías y afines con énfasis en ingredientes naturales para la industria cosmética en Colombia. Informe final. Análisis de desempeño implementación de las herramientas del sistema de inteligencia tecnológica análisis prospectivo. Bogotá, 147 p. 2009. Disponible En: http://www.agronet.gov.co/www/docs agronet/2009713162330 Prospectiva a gronet 2009.pdf 
27. Organización del Tratado de Cooperación Amazónica, OTCA. Impactos actuales y potenciales de las enfermedades de los cultivos perennes de la Amazonía y posibilidades de control para el desarrollo sostenible de la región. Tratado de cooperación Amazónica Secretaría pro tempore Caracas, Venezuela, p 91. 1999. Recuperado 23 de septiembre de 2010. Disponible En: http://www.otca.org.br/publicacao/SPT-TCA-VEN-70bw.pdf

28. Pérez L., Martínez G. La Bixa orellana en el tratamiento de afecciones estomatológicas, un tema aún por estudiar: Revista Cubana de Farmacia, 44 (2). 2010.2 Disponible En: http://scielo.sld.cu/scielo.php?pib=S003475172010000200012\&script=sci artte $\underline{x t}$

29. Pérez M., Albacerra R. Al Achiote Bixa Orellana. Boletín bimestral de la Comisión Nacional para el conocimiento y uso de la diversidad. 7 (46): 7. 2003.

30. Pérez O., Rojas A. Cinética y extracción de colorantes naturales. Universidad de las Américas. 2010.2 Disponible En: http://www.conocimientotextil.com/2010/05/cinetica-y-extraccion-decolorantes.html.

31. Pérez S., Cuen M., Becerra R. El achiote. Conabio Rev. Biodiversitas, 46: 711. 2003

32. Perú ecológico. Flora del Perú. Ficha técnica del cultivo del achiote. Perú. Recuperado 15 de diciembre de 2010. Disponible En: http://peruecologico.com.pe/flo achiote 1.html

33. Productos naturales peruanos Perú Cóndor. Recuperado 23 de Septiembre de 2010. Disponible En: http://www.perucondor.com/artículos/es achiote01.html

34. Sahaza D. El achiote (Bixa orellana L). Universidad Nacional de Medellín. 2001. Recuperado 3 de marzo de 2011. Disponible En: http://www.unalmed.edu.co/ crsequed/ACHIOTE.html

35. Toledo de Oliveira T., Nagem T., Rocha da Costa M., Marciano da Costa L., Magalaes L., Stringheta E., Biological properties of natural dyes. Ars Pharmaceut. 45 (1): 5-20. 2004.

36. Valero J. J. El cultivo del achiote. Universidad Francisco de Paula Santander, Facultad de Ciencias Agrarias, 2009. Recuperado 23 de noviembre de 2010. http://tibuyanito.blogspot.com/2009/03/el-cultivo-de-achiote-bixa-orellana-l.html

37. Vallejo F. Evaluación de germoplasma de achiote Bixa orellana L.; estudios básicos sobre asociaciones fenotípicas y biología floral. Universidad Nacional Sede Palmira. p 17. Recuperado 15 de enero 2011. Disponible En: http://www.revistas.unal.edu.co/index.php/actaagronómica/article/.../16260

38. Vonthron C, Weniger B, Ouattara M, Bi F, Kamenan A, Lobstein A et al. In vitro antiplasmodial activity and cytotoxicity of ethnobotanically selected Ivorian plants. Journal Ethnopharmacol. 87 (2-3): 221-225. 2003. 